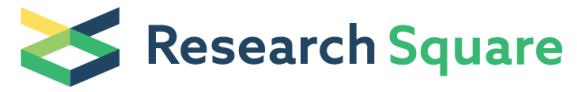 \\ Preprints are preliminary reports that have not undergone peer review. \\ They should not be considered conclusive, used to inform clinical practice, or referenced by the media as validated information.
}

\section{Primary Undifferentiated Pleomorphic Sarcoma in Oral-maxillary Area: Retrospective Study and Molecular Analysis}

\section{Meng Xiao}

Department of Oral and Maxillofacial-Head and Neck Oncology, Shanghai Ninth People's Hospital, Shanghai Jiao Tong University, School of Medicine, Shanghai 200011,China.

\section{Yuanhe You}

Department of Oral and Maxillofacial-Head and Neck Oncology, Shanghai Ninth People's Hospital, Shanghai Jiao Tong University, School of Medicine, Shanghai 200011,China

\section{Zhong Du}

Department of Oral and Maxillofacial-Head and Neck Oncology, Shanghai Ninth People's Hospital, Shanghai Jiao Tong University, School of Medicine, Shanghai 200011,China.

\section{Zhuowei Tian}

Department of Oral and Maxillofacial-Head and Neck Oncology, Shanghai Ninth People's Hospital, Shanghai Jiao Tong University, School of Medicine, Shanghai 200011,China.

\section{Guisong Xu}

Department of Oral and Maxillofacial-Head and Neck Oncology, Shanghai Ninth People's Hospital, Shanghai Jiao Tong University, School of Medicine, Shanghai 200011,China.

\section{Rongxin Shi}

Shandong University Stomatology Hospital

\section{Lizhen Wang}

Department of Oral Pathology, Shanghai Ninth People's Hospital, Shanghai Jiao Tong University, School of Medicine, Shanghai 200011,China

\section{Yanan Wang ( $\nabla$ shore70001@126.com )}

Shagnhai 9th people's hospital

\section{Research}

Keywords: Undifferentiated pleomorphic sarcoma, oral-maxillary area, retrospective study, whole exonic sequencing

Posted Date: November 9th, 2020

DOI: https://doi.org/10.21203/rs.3.rs-103177/v1 
License: (c) (i) This work is licensed under a Creative Commons Attribution 4.0 International License. Read Full License 


\section{Primary undifferentiated pleomorphic sarcoma in oral-maxillary area: retrospective study and molecular analysis}

Meng Xiao ${ }^{1,2,4 \#}$, Yuanhe You ${ }^{1,2,4 \#}$, Zhong $\mathrm{Du}^{1,2,4}$, Zhuowei Tian ${ }^{1,2,4}$, Guisong $\mathrm{Xu}^{1,2,}$

${ }^{4}$, Rongxin Shi ${ }^{*}$, Lizhen Wang ${ }^{1,3,4^{*}}$, Yan'an Wang ${ }^{1,2,4 *}$

1 National Clinical Research Center for Oral Disease, Shanghai, 200011, China.

2 Department of Oral and Maxillofacial-Head and Neck Oncology, Shanghai Ninth

People's Hospital, Shanghai Jiao Tong University, School of Medicine, Shanghai 200011,China.

3 Department of Oral Pathology, Shanghai Ninth People's Hospital, Shanghai Jiao

Tong University, School of Medicine, Shanghai 200011,China.

4 Shanghai Key Laboratory of Stomatology and Shanghai Research Institute of

Stomatology, Shanghai 200011, China

5 Department of stomatology, Tengzhou Central People's Hospital, Tengzhou 277500,

Shandong Province, China

\# The first two authors contributed equally to this paper.

*Correspondence to: Dr. Yan'an Wang, Dr. Lizhen Wang, and Dr. Rongxin Shi Shanghai Ninth People's Hospital, Shanghai Jiao Tong University, School of Medicine, Shanghai 200011, China. Tel: +86-21-53591389; e-mail: wangya1982@sh9hospital.org;shore70001@126.com; lizhenwang535@ hotmail.com; srxsdtz@126.com 


\section{Abstract}

Background: Undifferentiated pleomorphic sarcoma (UPS) in oral-maxillary area is rarely reported. Herein, we aimed to investigate the clinical characteristics, treatment strategies, prognosis, and molecular features of the oral-maxillary UPS.

Methods: Retrospectively, we reviewed the UPS patients who were diagnosed and treated in our department. The medical histories, imaging features, histopathological characteristics, treatment strategies, clinical outcomes were summarized and analyzed. Besides, the molecular features were demonstrated by whole exonic sequencing.

Results: Totally, 10 cases with primary oral-maxillary UPS were included. The rapidly progressive UPS can easily develop at advanced and life-threatening stage, especially concerning the complex anatomical structures and spaces in the oral-maxillary area. The finial diagnosis for UPS greatly depends on histological findings and immunohistochemistry under the exclusion of all the possible differential diagnosis. Retrospectively, the treatment strategies for the involved cases still referred to those of oral squamous cell carcinoma. Statistically, the median overall survival (OS) for all the included cases was 7.75 months (range: 5-17 months). Comparatively, 3 cases had improved OS (median survival: 17 months, range: 17-18 months) experienced PR/SD with neoadjuvant chemotherapy (anlotinib). Cancer driver genes detection revealed GBP4 as a candidate driver gene for the oral-maxillary UPS. Additionally, a missense mutation in gene PIK3CA (p.E545K) was also identified.

Conclusion: Our findings can greatly expand the knowledge about the oral-maxillary 
UPS, and provide molecular evidences to improve the therapeutic options for the oral-maxillary UPS. Further studies are warranted to validate our discoveries on the oral-maxillary UPS.

Key words: Undifferentiated pleomorphic sarcoma; oral-maxillary area; retrospective study; whole exonic sequencing 


\section{Introduction}

Undifferentiated pleomorphic sarcoma (UPS) has been established as an entity in the WHO-classification of soft tissue sarcoma since 2002[1-3]. UPS is very rare and constitutes less than $5 \%$ of all sarcomas in adults, most of which occurs in the extremities and the trunk of elderly patients $[4,5]$. The etiology for UPS is unknown, and the clinical features of UPS are not specific [6]. Till now, the clinical characteristics and therapeutic experiences of primary UPS are limited, and patients suffered from UPS experience a poor prognosis [7, 8].

Accordingly, head and neck localization of UPS is rare, with an incidence ranging from $4 \%$ to $10 \%$ [9]. The most frequent involved sites for UPS in the head and neck area are neck and parotid, followed by scalp, face, anterior skull base and orbit [9]. Primary UPS in the oral-maxillary area is exceedingly rare. Anatomically, the oral-maxillary area is a key site in head and neck. Clinical practice indicates that the outcomes for the patients with oral-maxillary are extremely poor. So, it is necessary to get to know the oral-maxillary UPS. In this study, we managed to collect the primary oral-maxillary UPS treated in our department, retrospectively. The clinicopathological characteristics and the improved treatment strategies for the involved primary UPS were summarized and analyzed. Besides, the molecular features for the primary oral-maxillary UPS were preliminarily demonstrated by whole exonic sequencing (WES). 


\section{Patients and methods}

\section{Patients}

Retrospectively, we reviewed and collected the UPS patients who were pathologically diagnosed and experienced combined treatment between 2015 and 2020 at the Department of Oral Maxillofacial-Head and Neck Oncology, Shanghai Ninth People's Hospital. To be included in this study, the following criteria were met for each case: (a) primary/recurrent UPS in the oral-maxillary area; (b) intact medical histories of diagnosis and treatments; (c) intact histopathological data; (d) intact imagelogical records; (e) follow-up information as complete as possible. The following cases were excluded: (a) the metastasized/secondary UPS involved the oral-maxillary areas; (b) patients with only histopathological biopsy and without subsequent treatments. This study was approved by the Medical Ethics Committee of the Ninth People's Hospital, Shanghai Jiao Tong University School of Medicine (SH9H-2020-T321-1).

The demographic data of the involved patients, including sex, age, primary tumor site, clinical presentation, and previous radiation history, were summarized. The TNM staging for each involved case referred to the American Joint Committee on Cancer (AJCC) Cancer Staging manual for head and neck cancer ( $8^{\text {th }}$ Edition, 2017). The clinical outcomes for each case were collected and summarized retrospectively from the follow-up information.

\section{Imaging examinations}

All patients experienced enhanced computed tomography (CT) and/or magnetic resonance imaging (MRI) examinations from the skull base to the clavicular level at 
their primary visit and during follow-up. Chest CT imaging was also performed for each case at the first visit and during follow-up. Representative CT/MRI images were downloaded from the Hospital Information System (HIS) of the hospital.

\section{Microscopic observations}

The final pathological reports were presented based on pathologic examinations by using haematoxylin-eosin (HE) and immunohistochemical (IHC) staining. IHC staining was performed to confirm the diagnosis of UPS. For IHC staining, a routine panel for the diagnosis of malignant tumors was used. IHC was performed with primary antibodies against AE1/AE3 (pancytokeratin), $\mathrm{CKH}$ (Cytokeratin-high molecular weight), EMA (Epithelial membrane antigen), Vimentin, ki67, INI1 (Integrase interactor 1), CD31, and ERG (v-ets erythroblastosis virus E26 oncogene homolog) (Autostainer/Autostainer Plus, DAKO, Denmark).

\section{Whole Exonic Sequencing and Analysis}

Genomic DNA was extrected via QIAamp DNA FFPE Tissue Kit (QIAGEN, Germany). Gene Exonic regions were seclected by Agilent SureSelect Human All Exon V6 Kit and library generated according to manufacturer's instruction (Agilent Technology, USA). DNA library was sequenced on Illumina NovaSeq platform via PE150 protocol (Illumina, USA). About 30G Raw data (300x in depth) were trimmed and mapped to reference genome hg38 using Burrows-Wheeler Aligner (BWA, http://bio-bwa.sourceforge.net/) tool. PCR duplicates were removed using Picard (http://github.com/broadinstitute/picard). Somatic SNP and Indel were discovered according to Genome Analysis Toolkit (GATK, http://software.broadinstitute.org/gatk/) 
workflow and annotated using ANNOVAR software with Refseq, CLINVAR, COSMIC, dbSNP150 database. Cancer driver genes detection was performed by maftools oncodrive function which based on oncodrive CLUST algorithm (http://bitbucket.org/bbglab/oncodriveclust). Structure variants detection was performed by delly2 and annotated using ANNOVAR with DGV database (http://dgv.tcag.ca). Factera (https://factera.stanford.edu/) was used to detect the gene fusion in the genome. Control-Freec (http://boevalab.com/FREEC/) was used to detect the copy number variation. Microsatellite Instability (MSI) was detected by MSIsensor2 (https://github.com/niu-lab/msisensor2).

\section{Statistical analysis}

Statistical analyses were conducted using IBM SPSS Statistics version 20.0 software (IBM Corp., Armonk, NY, USA). The overall survival for the included cases was analyzed by Kaplan-Meier method. A p-value $<0.05$ was considered statistically significant.

\section{Results}

\section{Clinical characteristics of the involved UPS patients}

As shown in Table 1, of the 10 involved UPS patients, 5 patients were male (median age at diagnosis 46.8 years, range 39-59 years), and 5 patients were female (median age at diagnosis 49.6 years, range 20-79 years). Regarding the primary sites of origin included the gingiva (4/10), tongue (3/10), buccal mucosa (1/10), palate $(1 / 10)$, and maxillary sinus (1/10). Clinically, the most common symptom was the presentation of a rapidly and widely progressive soft-tissue mass. At the time of primary diagnosis, all patients developed tumors at the T3/T4 stage. In addition, no lymph node metastasis or distal metastasis was detected in any of the cases at their primary 
diagnosis. Based on the medical history of the involved patients, 2 patients had records of OSCC history with previous radiation therapy.

\section{Imaging characteristics of the UPS in oral-maxillary area}

As summarized in Table 1, the complex structures of the oral-maxillofacial area make the wide invasion of UPS. Imageological diagnosis based on CT and/or MRI was necessary pre-operation. During the preoperative radiological examination, enhanced CT demonstrated a solid mass with malignant characteristics (Figure 1). Based on the CT images of the included cases, we observe an extensive invasion of UPS to the hard/soft palate, nasal cavity, maxillary sinus, pterygopalatine fossa, skull base, orbit, parapharyngeal area, mandible, masticatory muscles, mouth floor, tongue base, oropharyngeal area, and carotid sheath (Figure 1A-E). As shown in Figure 1F-H, T2WI MRI and contrast-enhanced MRI confirmed a solid mass with heterogeneous signals and heterogeneous enhancement. Besides, the CT/MRI did not indicate any lymph node metastasis. According to the aggressive clinical symptoms and CT/MRI findings, clinicians could recognize this soft-tissue mass as malignant tumor preoperatively.

\section{Histopathological characteristics of the UPS}

The finial diagnosis for UPS greatly depends on histological findings and immunohistochemistry (IHC). The microscopic examination of HE-stained sections revealed a malignant neoplasm with numerous spindle-shaped fibroblasts intermingled in fascicles (Figure 2A). In high powered view, varying amounts of nuclear pleomorphism could be observed (Figure 2B). IHC analysis demonstrated vimentin positivity (Figure $2 \mathrm{~F}$ ), and it was negative for the epithelial markers, such as 
AE1/AE3, CKH, EMA (Figure 2C-E). Besides, high Ki-67 activity was widely observed (Figure 2G). Negative INI1 staining excluded the possibility of malignant peripheral nerve sheath tumor (Figure 2H). Notably, positive CD31 and ERG staining was observed in 5/10 patients (Figure 2I, J). Under the exclusion of all the possible differential diagnosis, the involved cases were eventually diagnosed as UPS.

\section{Treatment and prognosis of the UPS in oral-maxillary area}

Till now, the therapeutic strategies for the UPS in oral-maxillary area have not reached a consensus. Retrospectively, the treatment strategies for the involved cases still referred to those of oral squamous cell carcinoma (OSCC) in our department. The treatment strategies and prognosis outcomes for all the included cases were summarized in Table 2. Five patients accepted neoadjuvant chemotherapy before operation, and 1 of them achieved partial response (PR) for anlotinib. A total of 10 patients underwent extensive resection of the mass (pathological confirmation for the resection margins as $\mathrm{R} 0$ ), and 2 of them combined with one-stage neck dissection. Soft-tissue reconstructions (ALTF/PMMF) were performed in 8 cases. Postoperatively, 6 patients were treated with adjuvant radiotherapy and chemotherapy/target therapy. In addition, cases 2 received adjuvant chemotherapy, and cases 4 received adjuvant radiotherapy only. Case 1 and case 6 did not accepted adjuvant therapy postoperatively.

Generally, the outcomes for the UPS in head and neck were not encouraging. Statistically, the median survival for all the included cases was 7.75 months (range: 5-17 months) (Figure 3A). Three cases had improved OS (median survival: 17 months, 
range: $17-18$ months) experienced $\mathrm{PR} / \mathrm{SD}$ with neoadjuvant chemotherapy (anlotinib) and adjuvant radiotherapy/chemotherapy compared with other cases (median survival: 6.25 months, range: 5-12 months; $p=0.0101$; Figure 3B). Seven cases died from the recurrence of the UPS in head and neck, and 2 cases died from lung metastasis and the subsequent malignant pleural effusions (Figure 3C-D). In addition, 1 case died from recurrence and metastasis (Table 2).

\section{Molecular features of the UPS in oral-maxillary area}

In this study, we described a rapidly progressive case of UPS originated from the buccal area (responded poorly to neoadjuvant chemotherapy and died from lung metastasis, Figure 4A) and analyzed its mutation variations (Figure 4B). Totally, we detected 739,096 SNVS (single nucleotide variants), 113,934 INDELs (insertion or deletion), 27, 514 SVs (structure variant), and 90 gene fusions (Figure 4B). Cancer driver genes detection revealed GBP4 as a candidate driver gene for the oral-maxillary UPS (Figure 4C). A total of 8 missense mutations were detected in the exon 10 of the gene GPB4, including rs561042, rs561037, rs608339, rs1142890, rs1142889, rs1142888, rs1142886, and rs655260 (Figure 4D). Besides, we also identified a missense mutation in gene PIK3CA (rs104886003, Figure 4E). The WES data revealed a high TMB (tumor mutation burden, 152.01) in the detected case (Figure 4F). Comparatively, the MSI (microsatellite instability) rate of the case was detected as MSI-L/MSS (microsatelite instability-low/microsatelite instability-stable, Figure 4G). 


\section{Discussion}

In this study, we managed to demonstrate the clinicopathological characteristics and molecular features of UPS in the oral-maxillary area for the first time. The rapidly progressive UPS can easily develop at advanced and life-threatening stage (wide invasion of soft tissues and bones), especially concerning the complex anatomical structures and spaces in the oral-maxillary area. Comparatively, regional lymph metastasis of UPS is rarely observed. The histopathological diagnosis for UPS greatly depends on the histological examination and extensive immunohistochemical markers to differentiate it from other malignant epithelial and mesenchymal tumors [4]. The main differential diagnoses that must be considered and excluded before the eventual diagnosis of UPS are poorly differentiated carcinoma, melanoma, dedifferentiated liposarcoma, pleomorphic liposarcoma, pleomorphic leiomyosarcoma, myxofibrosarcoma, pleomorphic rhabdomyosarcoma, and malignant peripheral nerve sheath tumor $[6,9]$. Previous studies have demonstrated that UPS is the most common histologic subtype of radiation-associated sarcoma (RAS) [10, 11], and 2/10 of the included UPS patients had previous radiation-therapy history. So, radiation-induced UPS cannot be ignored for patients with radiation history in the oral-maxillary area.

Accordingly, surgical resection with negative resection margins is the mainstay of treatment for the UPS [7, 9]. In our department, radical resection with negative margins has been performed for the UPS patients. Neck dissection is not regular recommended for the rare rate of cervical metastasis of UPS patients. Compared with the UPS originated from other sites, the wide tissue-defects in the oral-maxillary area 
greatly affect the quality of life for the patients. In our department, Simultaneous soft-tissue flaps are recommended to reconstruct the composite tissue defects of the oral-maxillary system. Besides, treatment strategies for the oral-maxillary UPS have been developing through collaboration among clinicians within a multidisciplinary team (MDT) in our department. Concerning the limited data in literature, the roles of perioperative chemotherapy and radiation have been unclear [12, 13]. Herein, considering the poor prognosis of oral-maxillary UPS, MDT treatment strategy is recommended including neoadjuvant chemotherapy/targeted chemotherapy, radical surgery, adjuvant chemotherapy/targeted therapy, and adjuvant radiation. Comparatively, the prognosis of UPS arising from oral-maxillary area is much poorer when compared with UPS in other regions $[14,15]$. In this study, we also observed that the cases with better responses to the neoadjuvant chemotherapy (anlotinib) had improved OS, and IHC staining also indicated the positive expression of CD31 and ERG in some cases. What's more, further investigations are definitely warranted.

Recently, the molecular characterization of UPS originated from other tissues has been demonstrated sporadically $[16,17]$. Activation of Hedgehog and Notch signaling pathways have been demonstrated in cells with tumor-initiating potential from UPS [6]. Besides, the VGLL3 and YAP1 in the Hippo signaling pathway have been investigated to be overexpressed in a subset of UPS [6]. However, studies on the UPS mutational landscape are rarely conducted. This study is the first to use the WES to detect the gene mutation occurring in the oral-maxillary UPS. In the detected UPS case, cancer driver genes detection revealed GBP4 as a candidate driver gene. The 
GBPs are the members of the guanosine triphosphatase family (GTPases) that are induced by IFN $\gamma$ and have a role in resistance to pathogens [18]. A high expression of GBP1, GBP2, GBP3, GBP4 and GBP5 have been observed to be correlated to more favorable clinical outcomes in colorectal cancer, skin cutaneous melanoma, breast cancer, OSCC, esophageal squamous cell carcinoma, and Kaposi's sarcoma[19, 20]. The mutations of GBP4 might play key roles in the oncogenesis of oral-maxillary UPS, and more studies are deserved to investigate the roles of mutated GBP4 in the oncogenesis of UPS in detail. Additionally, a missense mutation in gene PIK3CA (p.E545K) was also identified. Till now, the mutational activation of the PI3K pathway has been demonstrated as a central event in many types of cancer [21]. Accordingly, PIK3CA E545K cells showed increased proliferation rates in all growth conditions compared with wild-type cells [21]. So, the mutation of PIK3CA might greatly contribute to the malignant progression of the oral-maxillary UPS [22]. The WES data revealed a high TMB (tumor mutation burden, 152.01) in the detected case, which has been emerged as a promising predictive biomarker for immune checkpoint inhibitor therapy [23].

In conclusion, our findings can greatly expand the knowledge about the mutation spectrum and oncogenesis of the oral-maxillary UPS, and provide molecular evidences to improve the therapeutic options for the UPS patients. Further studies are warranted to validate our discoveries on the oral-maxillary UPS.

\section{Acknowledgements}


Not applicable.

\section{Funding}

This work was partially supported by the National Natural Science Foundation of China [No. 81902746 and No. 81870780]. This work was partially sponsored by Shanghai Sailing Program [19YF1426800].

\section{Availability of data and materials}

The data sets generated and/or analyzed during the present study are available from the corresponding author on reasonable request.

\section{Author contributions}

Conceived and designed the analysis: Meng Xiao, Lizhen Wang, and Yan'an Wang; Collected the data: Yuanhe You, Zhong Du, and Zhuowei Tian; Contributed data or analysis tools: Zhuowei Tian and Guisong Xu; Performed the analysis: Meng Xiao and Yuanhe You; Wrote the paper: Meng Xiao, Lizhen Wang, and Yan'an Wang

\section{Ethics approval and consent to participate}

This study was approved by the Medical Ethics Committee of the Ninth People's Hospital, Shanghai Jiao Tong University School of Medicine [SH9H-2020-T321-1].

\section{Patient consent for publication}

Signed consent was obtained from all patients or their sibs included in the study

\section{Competing interests}

All the authors declare that they have no competing interests.

\section{References}

1. Müller-Richter UD, Kohlhof JK, Reichert TE, Roldán JC. Undifferentiated pleomorphic sarcoma of the orbital region. The British journal of oral \& 
maxillofacial surgery $2018 ; 46: 325-327$.

2. Fletcher CD, Vandenberg E, Molenaar W. Pleomorphic malignant fibrous histocytoma/undifferentiated high grade pleomorphic sarcoma. In: The WHO classification of tumors of soft tissue and bone. Lyon: IARC press 2002; p.120.

3. Fletcher C. The evolving classification of soft tissue tumours - an update based on the new 2013 WHO classification. Histopathology 2014; 64:2-11.

4. Srinivasamurthy BC, Kulandaivelu AR, Saha K, Saha A. Primary undifferentiated pleomorphic sarcoma of the breast in a young female: a case report. World journal of surgical oncology 2016; 14:186.

5. Moore CA, Kapila A. Undifferentiated Pleomorphic Sarcoma after Pirfenidone Use: A Case Report. The Permanente journal 2018; 22:17-116.

6. Widemann BC, Italiano A. Biology and Management of Undifferentiated Pleomorphic Sarcoma, Myxofibrosarcoma, and Malignant Peripheral Nerve Sheath Tumors: State of the Art and Perspectives. Journal of clinical oncology $2018 ; 36: 160-167$.

7. Yao D, Dai C. Clinical characteristics of the primary hepatic malignant fibrous histiocytoma in China: case report and review of the literature. World journal of surgical oncology 2012; 10:2.

8. Jeong CY, Kim CS. Undifferentiated pleomorphic sarcoma of the vocal fold. Ear, nose, \& throat journal 2016; 95:E12-e14.

9. Vuity D, Bogdan S, Csurgay K, Sapi Z, Nemeth Z. Malignant fibrous histiocytoma/undifferentiated high-grade pleomorphic sarcoma of the maxillary sinus: report of a case and review of the literature. Pathology oncology research 2013; 19:605-609.

10. Koyama T, Kobayashi T, Maruyama S, Abé T, Swelam WM, Kodama Y, Hoshina H, Takagi R, Hayashi T, Saku T. Radiation-induced undifferentiated high-grade pleomorphic sarcoma [malignant fibrous histiocytoma] of the mandible: report of a case arising in the background of long-standing osteomyelitis with a review of the literature. Pathology, research and practice 
$2014 ; 210: 1123-1129$.

11. Dineen SP, Roland CL, Feig R, May C, Zhou S, Demicco E, Sannaa GA, Ingram D, Wang WL, Ravi V, Guadagnolo A, Lev D, Pollock RE, Hunt K, Cormier J, Lazar A, Feig B, Torres KE. Radiation-Associated Undifferentiated Pleomorphic Sarcoma is Associated with Worse Clinical Outcomes than Sporadic Lesions. Annals of surgical oncology 2015; 22:3913-3920.

12. Spaulding Rt, Koumoundouros T, Parker JC. Metastatic undifferentiated pleomorphic sarcoma causing intraoperative stroke. Annals of clinical and laboratory science $2013 ; 43: 172-175$.

13. Soleymani T, Aasi SZ, Novoa R, Hollmig ST. Atypical Fibroxanthoma and Pleomorphic Dermal Sarcoma: Updates on Classification and Management. Dermatologic clinics 2019; 37:253-259.

14. Zhu Y, Hao D, Tang X, Sun L. Undifferentiated high-grade pleomorphic sarcoma of ethmoid sinus: a case report and literature review. Brazilian journal of otorhinolaryngology 2018; 84:389-392.

15. Sabesan T, Xuexi W, Yongfa Q, Pingzhang T, Ilankovan V. Malignant fibrous histiocytoma: outcome of tumours in the head and neck compared with those in the trunk and extremities. The British journal of oral \& maxillofacial surgery $2006 ; 44: 209-212$.

16. Li B, Li L, Li X, Wang Y, Xie Y, Liu C, Li F. Undifferentiated pleomorphic sarcoma with co-existence of KRAS/PIK3CA mutations. International journal of clinical and experimental pathology 2015; 8:8563-8567.

17. Lewin J: Identifying actionable variants using next generation sequencing in patients with a historical diagnosis of undifferentiated pleomorphic sarcoma. BMJ case reports 2018; 142:57-65.

18. Tyrkalska S, Candel S, Angosto D, Gómez-Abellán V, Martín-Sánchez F, García-Moreno D, Zapata-Pérez R, Sánchez-Ferrer Á, Sepulcre MP, Pelegrín P, Mulero V. Neutrophils mediate Salmonella Typhimurium clearance through the GBP4 inflammasome-dependent production of prostaglandins. Nature communications 2016; 7:12077. 
19. Britzen-Laurent N, Lipnik K, Ocker M, Naschberger E, Schellerer V, Croner R, Vieth M, Waldner M, Steinberg P, Hohenadl C, Stürzl M. GBP-1 acts as a tumor suppressor in colorectal cancer cells. Carcinogenesis 2013; 34:153-162.

20. Wang Q, Wang X, Liang Q, Wang S, Xiwen L, Pan F, Chen H, Li D. Distinct prognostic value of mRNA expression of guanylate-binding protein genes in skin cutaneous melanoma. Oncology letters 2018; 15:7914-7922.

21. Beaver JA, Gustin JP, Yi KH, Rajpurohit A, Thomas M, Gilbert SF, Rosen DM, Ho Park B, Lauring J. PIK3CA and AKT1 mutations have distinct effects on sensitivity to targeted pathway inhibitors in an isogenic luminal breast cancer model system. Clinical cancer research 2013; 19:5413-5422.

22. Kang S, Bader AG, Vogt PK. Phosphatidylinositol 3-kinase mutations identified in human cancer are oncogenic. Proceedings of the National Academy of Sciences of the United States of America 2005; 102:802-807.

23. Alborelli I, Bratic Hench I, Chijioke O, Prince S, Bubendorf L, Leuenberger L, Leuenberger LP, Tolnay M, Leonards K, Quagliata L, Jermann P, Matter MS. Robust assessment of tumor mutational burden in cytological specimens from lung cancer patients. Lung cancer 2020; 149:84-89. 
Table 1 Clinical features of 10 oral-maxillary UPS cases.

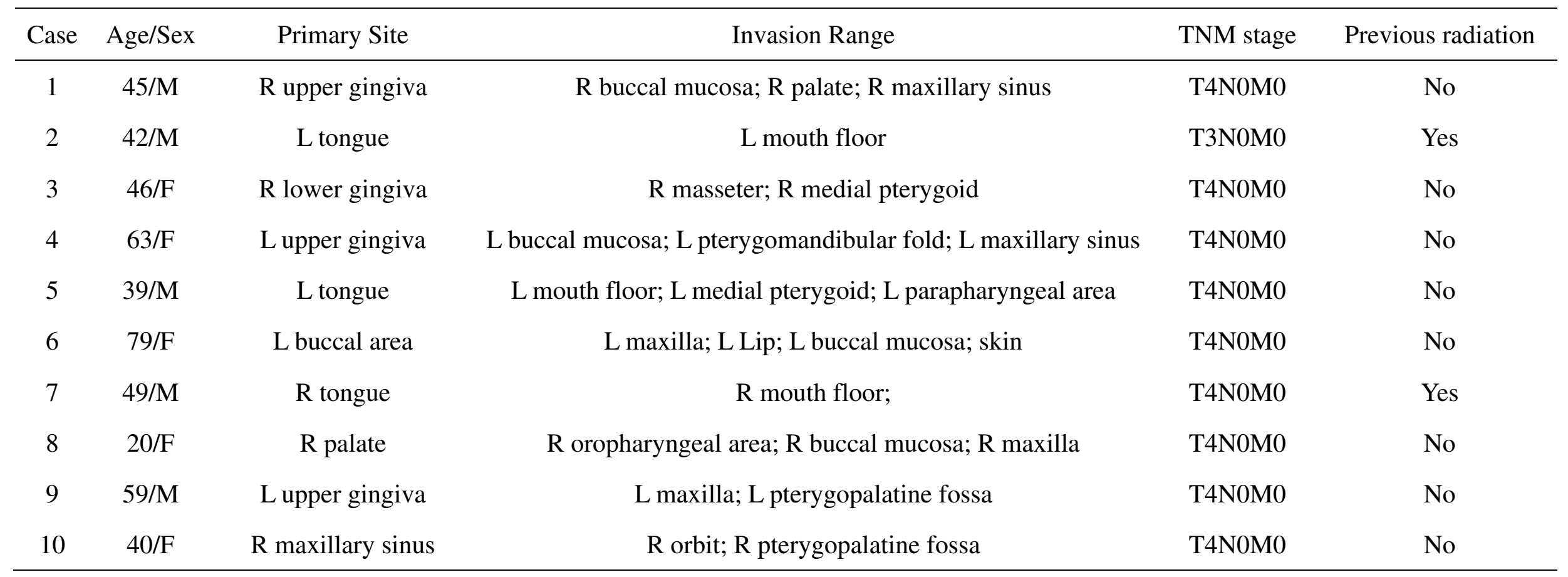

Note: M: male; F: female; R: right; L: left. 
Table 2 Treatment and clinical outcomes for the10 cases with oral-maxillary UPS.

\begin{tabular}{cccccc}
\hline Case & $\begin{array}{c}\text { Neoadjuvant } \\
\text { chemotherapy }\end{array}$ & Surgery & Radiation & $\begin{array}{c}\text { Chemotherapy/ } \\
\text { Target therapy }\end{array}$ \\
\hline 1 & No & Extensive Resection & No & No & Died at 9 m (Recurrence) \\
2 & No & Extensive Resection + SOND + ALTF & No & Yes & Die at $5 \mathrm{~m}$ (Recurrence) \\
3 & Anlotinib (PR) & Extensive Resection + ALTF & Yes & Yes & Die at $18 \mathrm{~m}$ (Metastasis) \\
4 & No & Extensive Resection & Yes & No & Die at $5 \mathrm{~m}$ (Recurrence) \\
5 & UN (PD) & Extensive Resection + RN+ PMMF & Yes & Yes & Die at $5 \mathrm{~m}$ (Recurrence) \\
6 & MAID (PD) & Extensive Resection + ALTF & No & No & Die at $6.5 \mathrm{~m}$ (Metastasis) \\
7 & No & Extensive Resection + ALTF & Yes & Yes & Die at $6 \mathrm{~m}$ (Recurrence) \\
8 & Anlotinib (SD) & Extensive Resection + ALTF & Yes & Yes & Die at $17 \mathrm{~m}$ (Recurrence) \\
9 & No & Extensive Resection + ALTF & Yes & Yes & Die at $12 \mathrm{~m}$ (Recurrence) \\
10 & Anlotinib (SD) & Extensive Resection + ALTF & Yes & Yes & Die at $17 \mathrm{~m}$ (Recurrence + Metastasis) \\
\hline
\end{tabular}

Note: PR: partial response; PD: progressive disease; SD: stable disease; UN: unknown; SOND: supraomohyoid neck dissection; RD: radical neck dissection; ALTF: Anterolateral thigh flap; PMMF: Pectoralis major myocutaneous flap. 

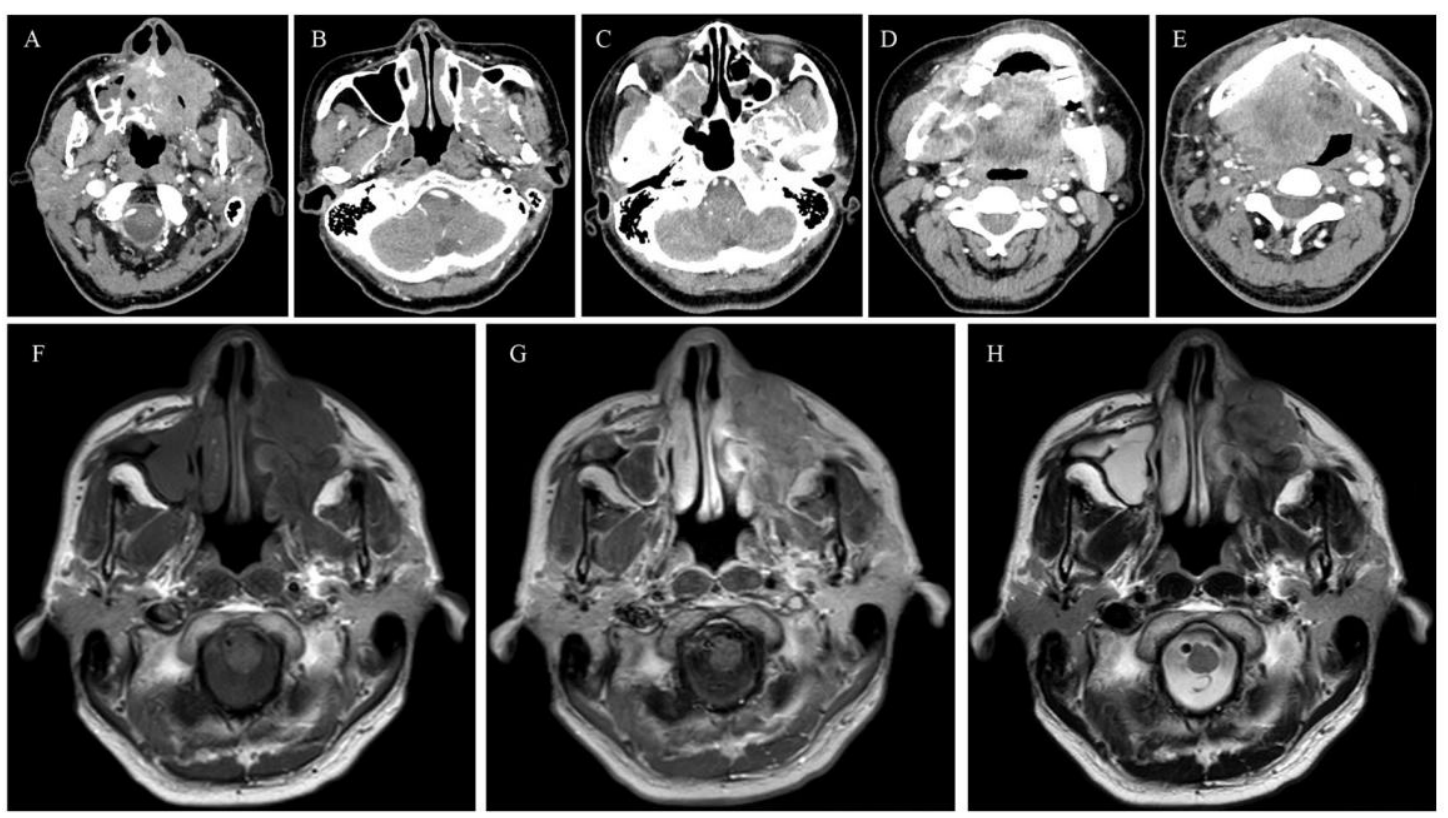

Figure 1: Representative CT/MRI images showing the local invasion of UPS in oral-maxillary area.(A-E) CT images: (A) Invasion of the hard/soft palate, nasal cavity, maxillary sinus, and skin; (B) Invasion of the maxilla and pterygopalatine fossa; (C) Invasion of the skull base and orbit; (D) Invasion of the parapharyngeal area, mandible, masticatory muscles, and mouth floor; (E) Invasion of the tongue base, mouth floor, oropharyngeal area, and carotid sheath. (F-H) MRI images: (F) T1WI; (G) Contrast-T1WI; (H) T2WI. 


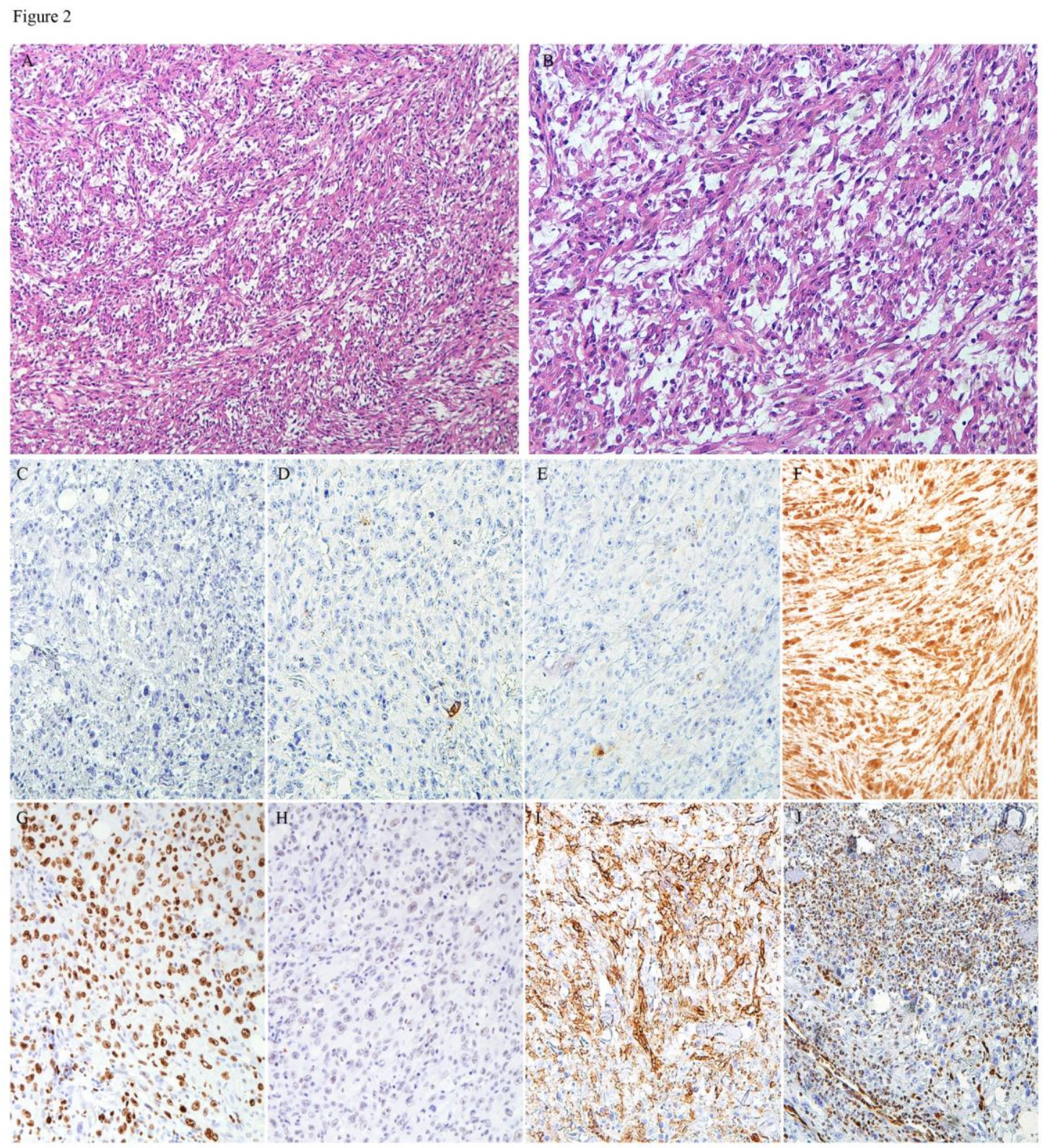

Figure 2: Representative histopathological images for the UPS in oral-maxillary area.

(A-B) H\&E stained sections: (A) 100x; (B) 200x. (C-J) Immunological characteristics:

(C) AE1/AE3; (D) CKH; (E) EMA; (F) Vimentin; (G) Ki67; (H) INI1; (I) CD31; (J)

ERG. 

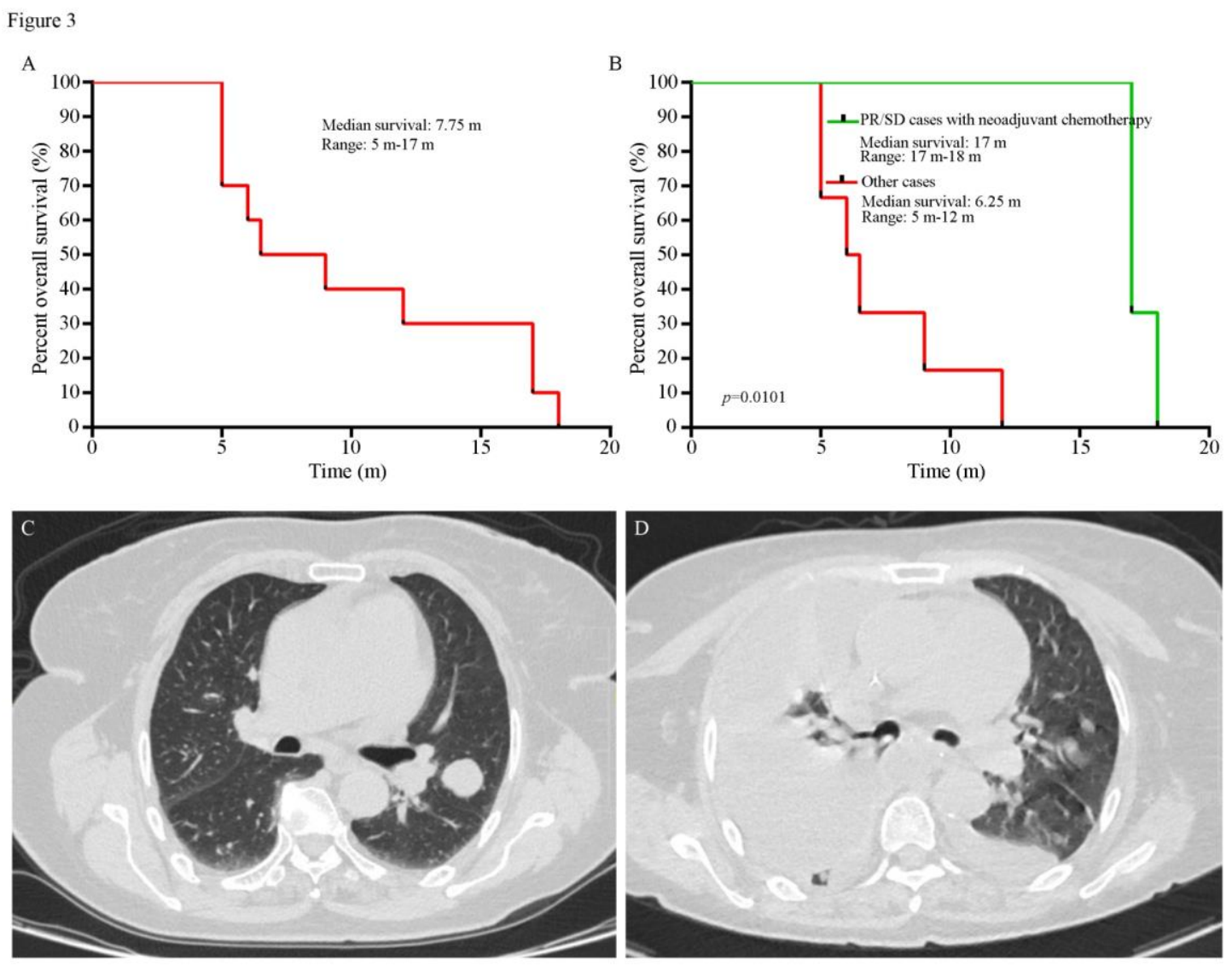

Figure 3: Prognosis analysis of the UPS cases in oral-maxillary area. (A) Analysis of overall survival for all the involved cases; (B) Analysis of overall survival stratified by the response of neoadjuvant chemotherapy; (C) Pulmonary nodules indicating lung metastasis; (D) Malignant pleural effusions resulted from the lung metastasis of the UPS. 


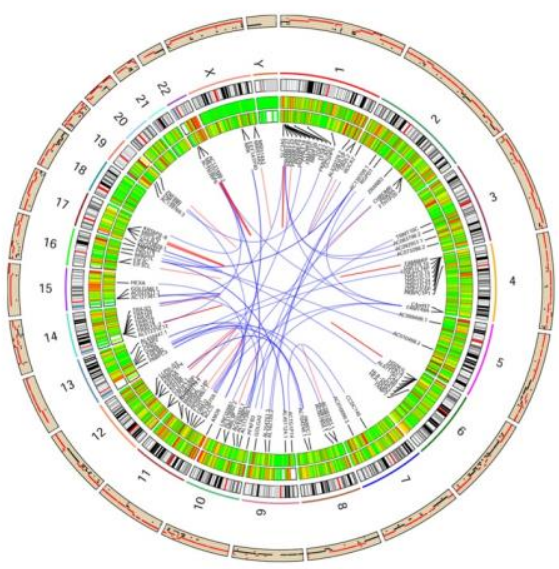

C
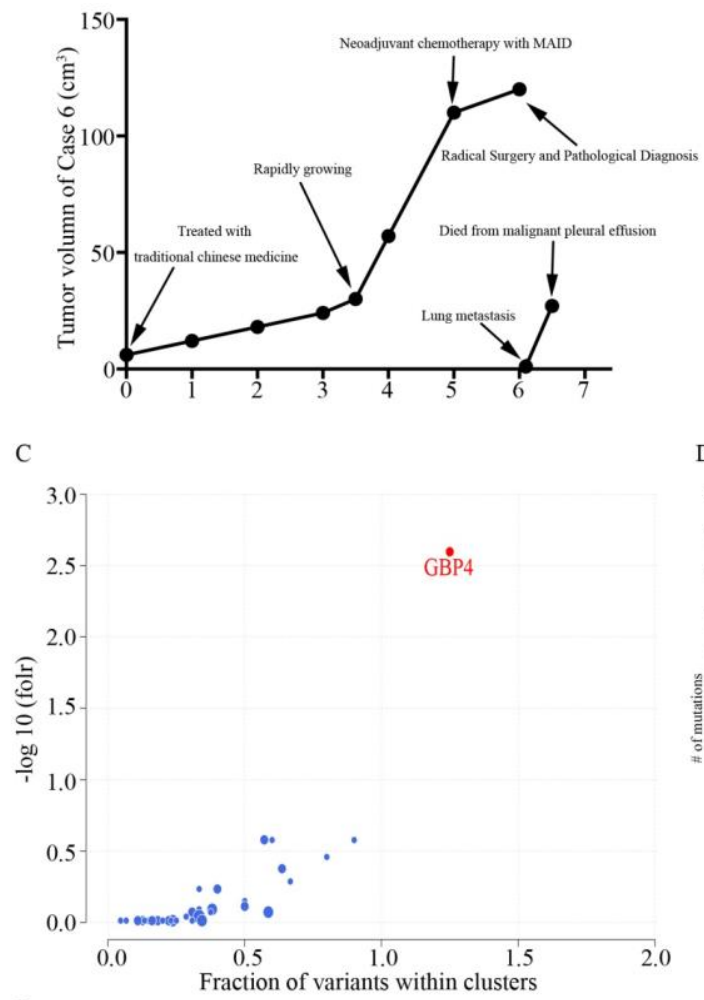

D

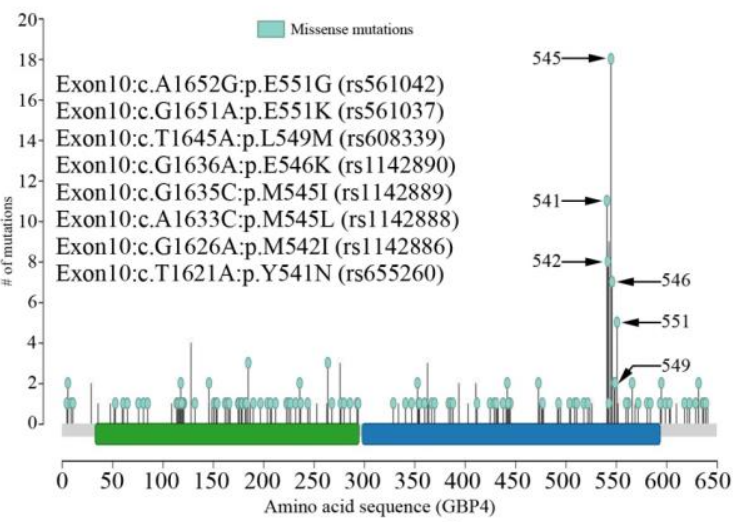

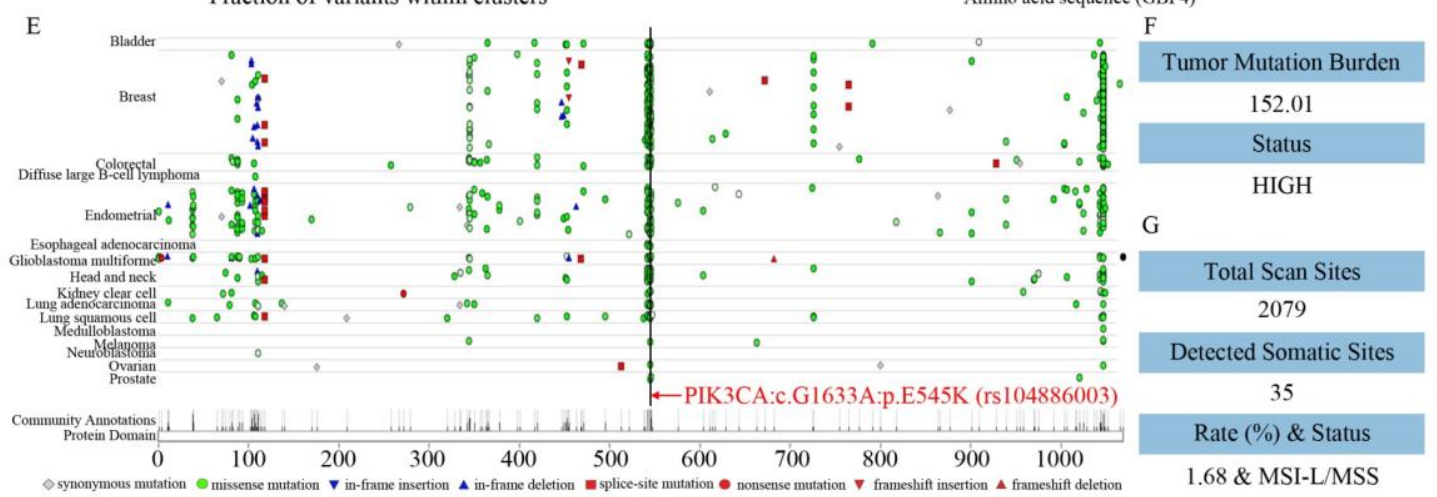

Figure 4: Medical process and molecular analysis for a UPS patient in oral-maxillary area. (A) Medical process for the detected case; (B) Circos plot showing genetic mutations detected by WES analysis; (C) GBP4 identified as cancer driver gene; (D) Location of the detected mutation sites for GBP4; (E) Location of the detected mutation site for PIK3CA; (F) Tumor mutation burden analysis; (G) Microsatellite instability analysis. 


\section{Figures}
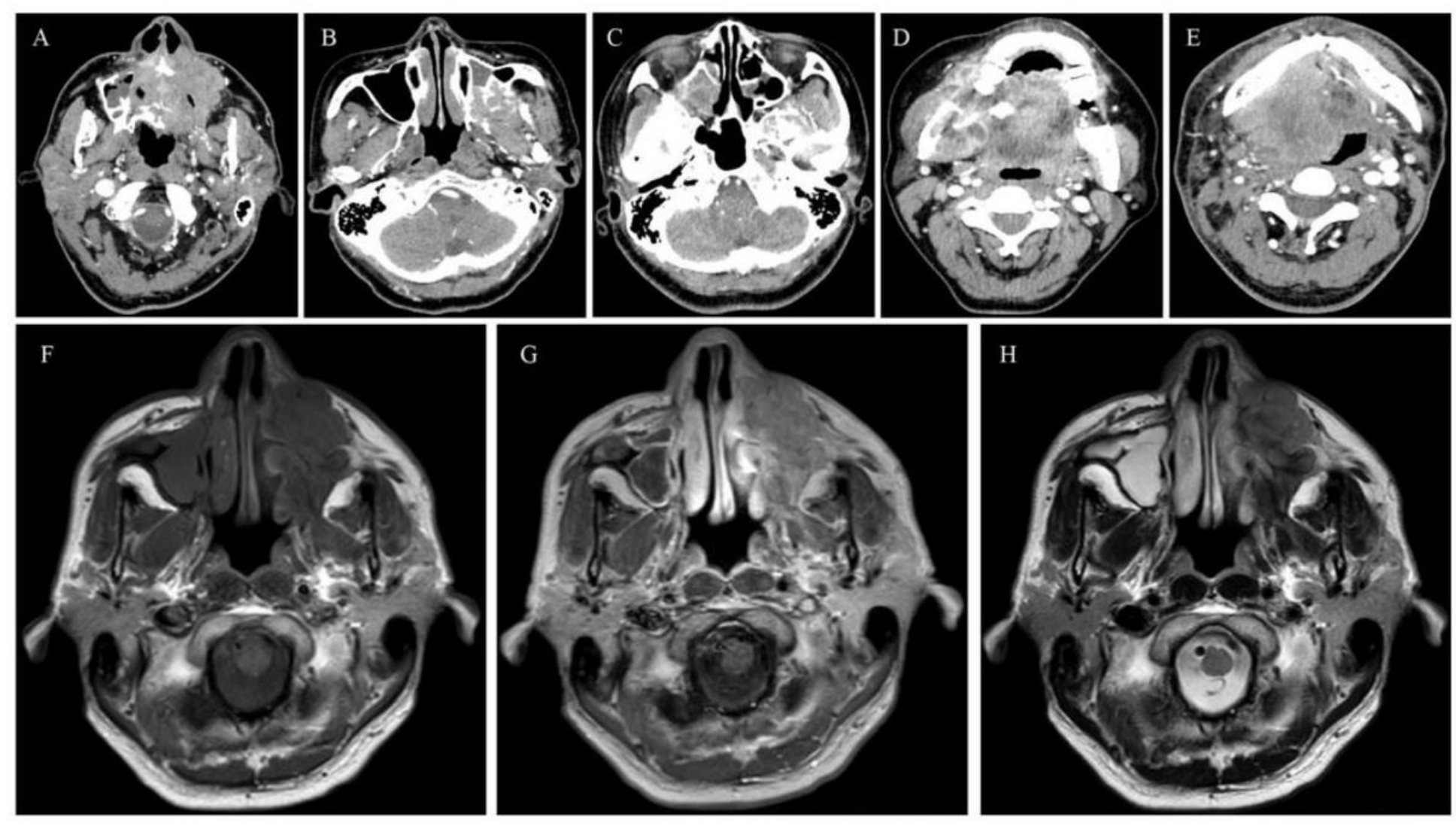

Figure 1

Representative CT/MRI images showing the local invasion of UPS in oral-maxillary area.(A-E) CT images: (A) Invasion of the hard/soft palate, nasal cavity, maxillary sinus, and skin; (B) Invasion of the maxilla and pterygopalatine fossa; (C) Invasion of the skull base and orbit; (D) Invasion of the parapharyngeal area, mandible, masticatory muscles, and mouth floor; (E) Invasion of the tongue base, mouth floor, oropharyngeal area, and carotid sheath. (F-H) MRI images: (F) T1Wl; (G) Contrast-T1WI; (H) T2WI. 


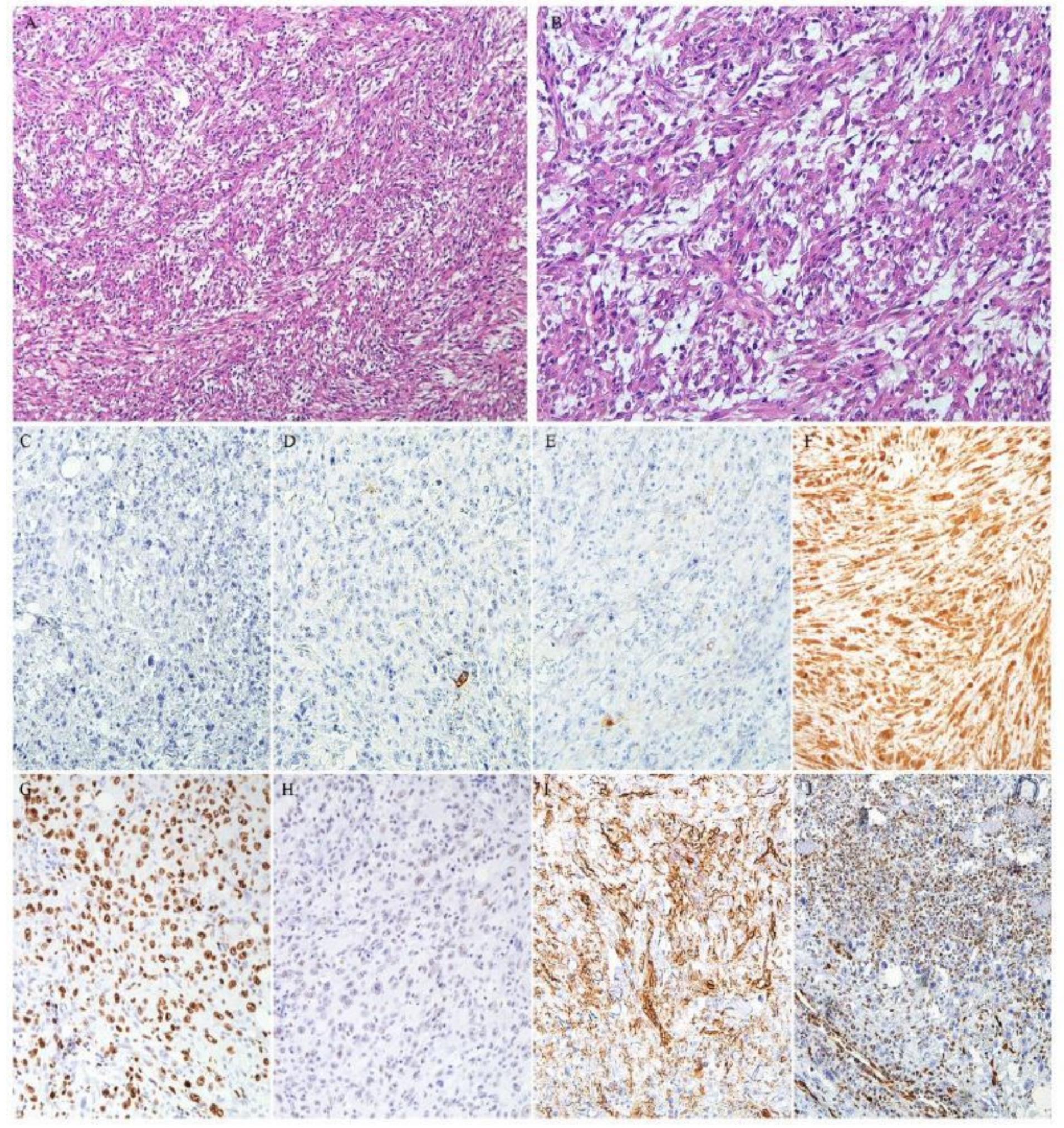

\section{Figure 2}

Representative histopathological images for the UPS in oral-maxillary area. (A-B) H\&E stained sections: (A) 100x; (B) 200x. (C-J) Immunological characteristics: (C) AE1/AE3; (D) CKH; (E) EMA; (F) Vimentin; (G) Ki67; (H) INI1; (I) CD31; (J) ERG. 

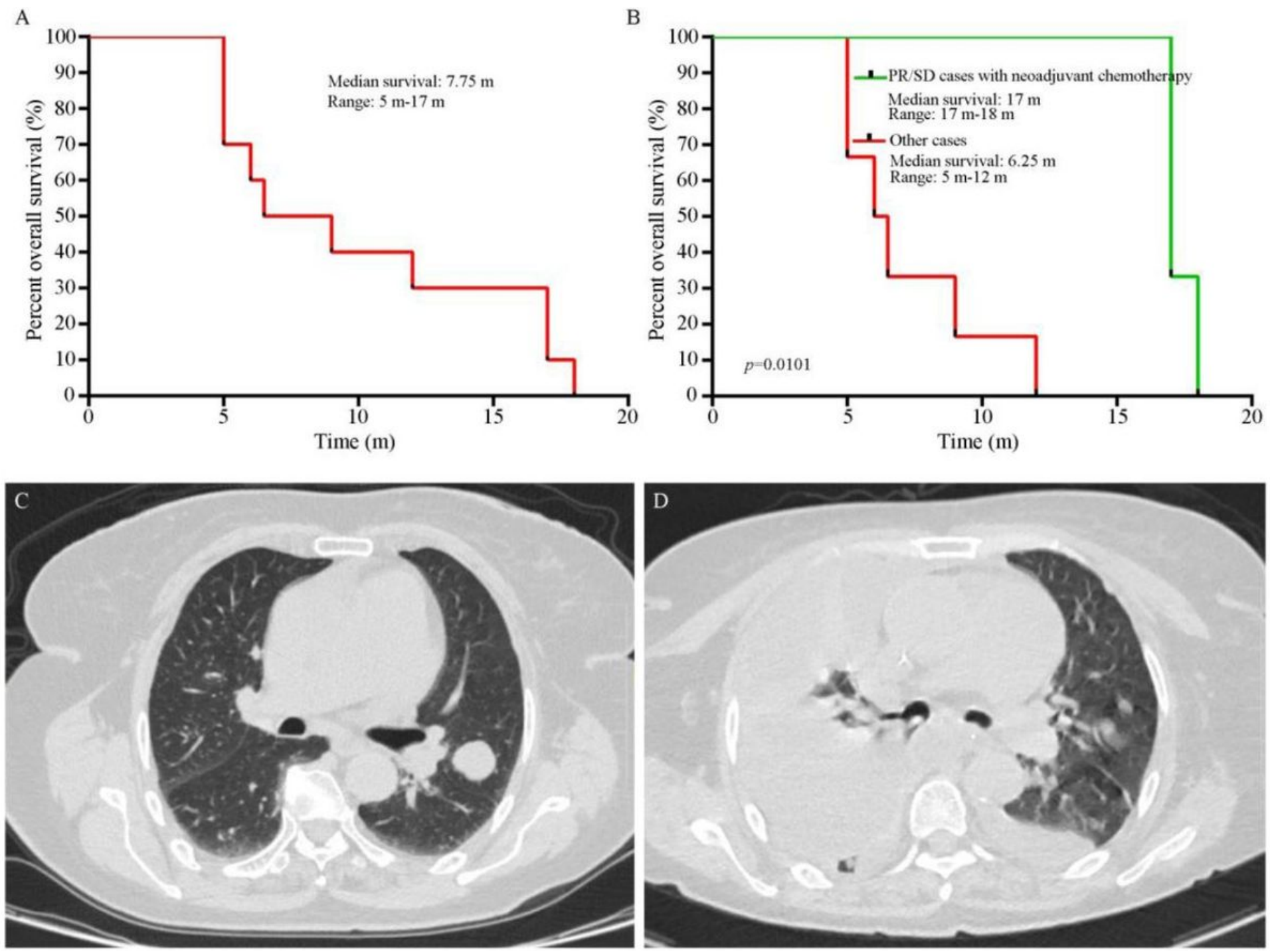

Figure 3

Prognosis analysis of the UPS cases in oral-maxillary area. (A) Analysis of overall survival for all the involved cases; (B) Analysis of overall survival stratified by the response of neoadjuvant chemotherapy; (C) Pulmonary nodules indicating lung metastasis; (D) Malignant pleural effusions resulted from the lung metastasis of the UPS. 
Figure 4

A

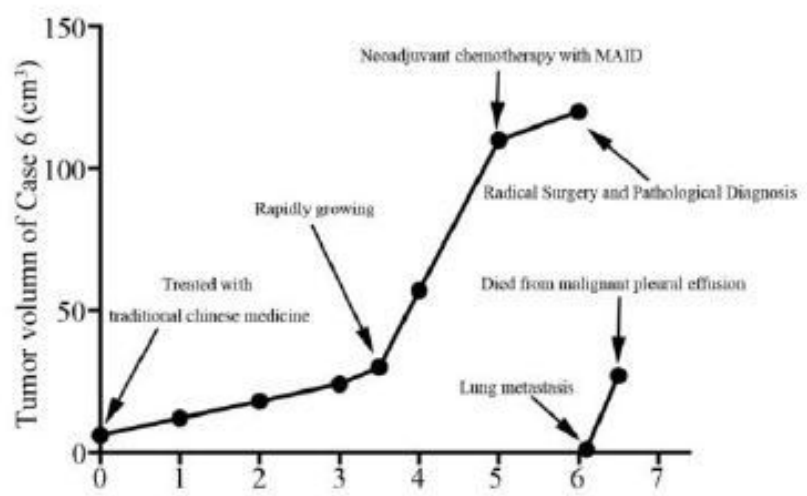

C

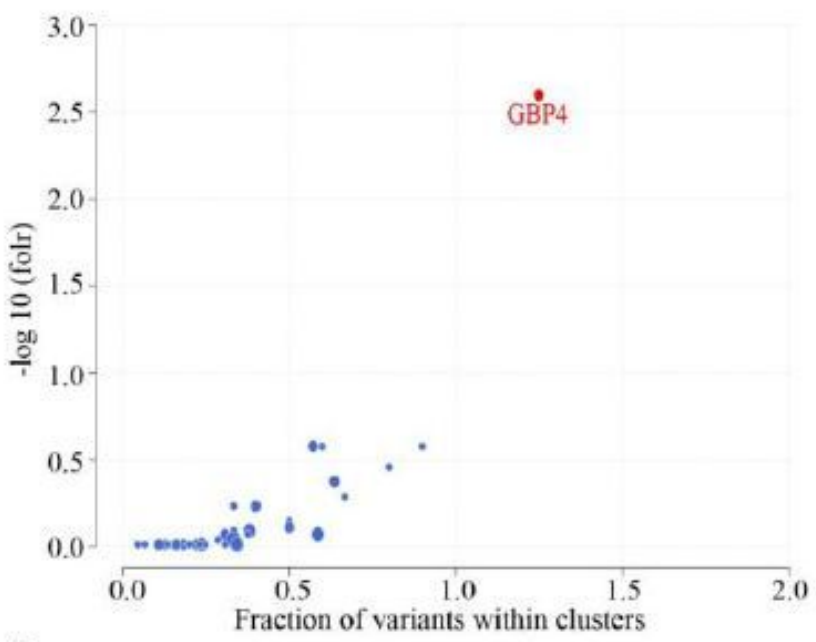

B

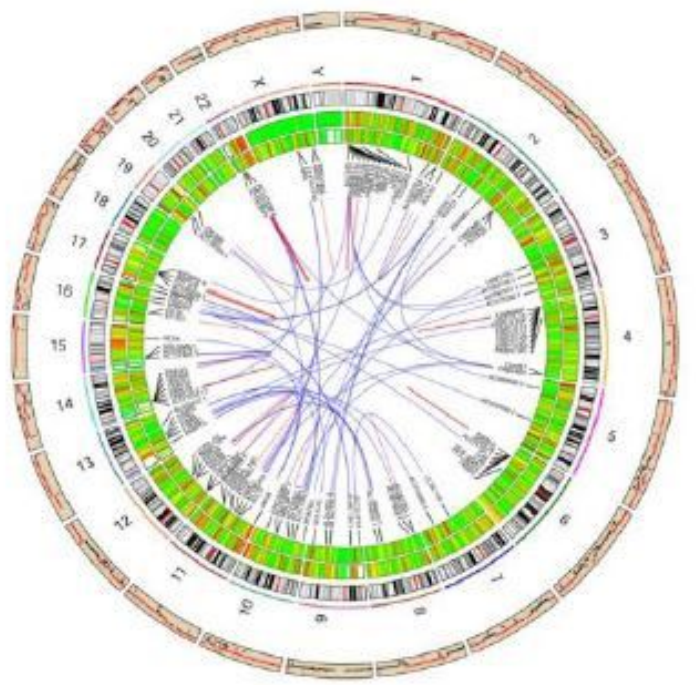

D

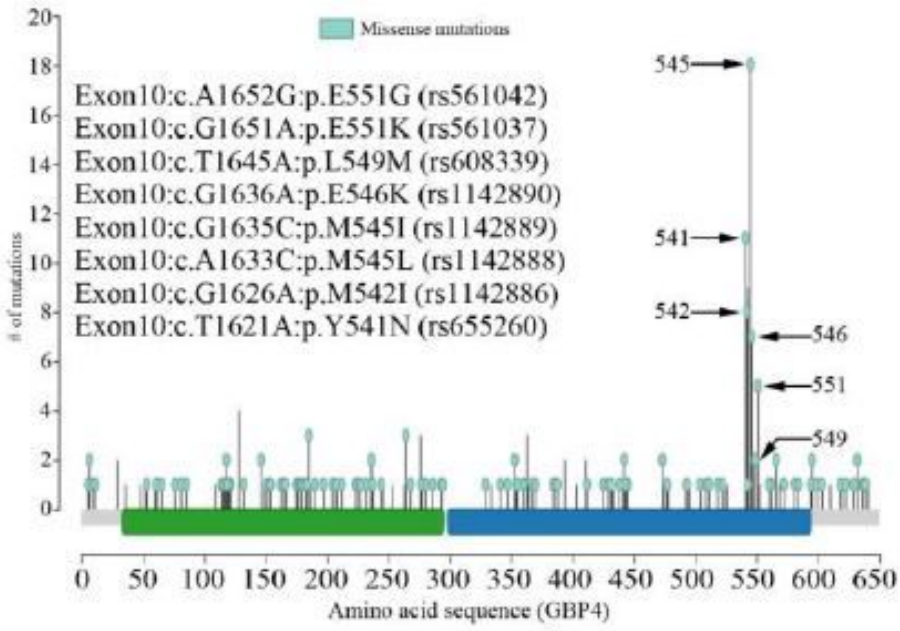

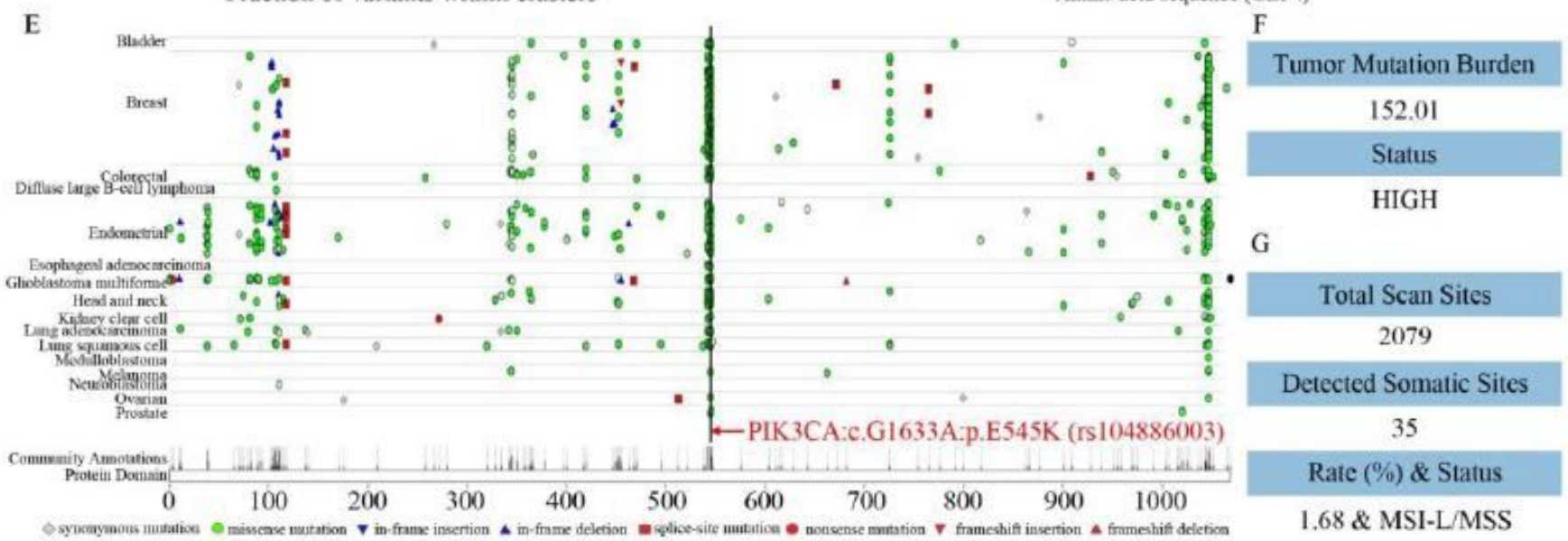

\section{Figure 4}

Medical process and molecular analysis for a UPS patient in oral-maxillary area. (A) Medical process for the detected case; (B) Circos plot showing genetic mutations detected by WES analysis; (C) GBP4 identified as cancer driver gene; (D) Location of the detected mutation sites for GBP4; (E) Location of the detected mutation site for PIK3CA; (F) Tumor mutation burden analysis; (G) Microsatellite instability analysis. 